\section{Arcobacter spp. in bovine milk: An emerging pathogen with potential zoonotic risk}

\author{
Marta Caruso, ${ }^{1,2}$ Laura Latorre, ${ }^{1}$ \\ Gianfranco Santagada, ${ }^{1}$ \\ Rosa Fraccalvieri, ${ }^{1}$ \\ Laura Maria Difato, ${ }^{1}$ \\ Angela Miccolupo, ${ }^{1}$ Loredana Capozzi, ${ }^{1}$ \\ Elisabetta Bonerba, ${ }^{3}$ Anna Mottola, ${ }^{3}$ \\ Antonio Parisi ${ }^{1}$ \\ ${ }^{1}$ Istituto Zooprofilattico Sperimentale \\ della Puglia e della Basilicata, Foggia; \\ ${ }^{2}$ Anthrax Reference Institute of Italy, \\ Istituto Zooprofilattico Sperimentale \\ della Puglia e della Basilicata, Foggia; \\ ${ }^{3}$ Department of Veterinary Medicine, \\ University of Bari, Italy
}

\begin{abstract}
The aim of the present study was to assess the prevalence and genetic characteristics of Arcobacter spp. in bovine bulk tank milk produced in Apulia Region (Italy). Samples collected from 396 dairy farms, after enrichment in a selective broth, were subjected to an Arcobacter genus specific Real Time PCR. Positive broths, previously filtered, were seeded on Karmali, MCCD and Columbia Blood Agar plates; presumptive Arcobacter spp. colonies were identified using an amplification and sequencing method and then characterized by Multi-Locus Sequence Typing (MLST). Prevalence of Arcobacter spp. in bovine milk samples was 5\% (20/396); A. butzleri was the only isolated species, in agreement with previous studies that reported $A$. butzleri as the most commonly recovered species in milk and dairy products. MLST analysis of the $20 \mathrm{~A}$. butzleri strains identified 81 alleles and 16 STs. Consistent with previous studies, MLST revealed a high level of heterogeneity between the $A$. butzleri isolates and confirmed the high discriminatory power of this method and its suitability for epidemiological investigations. This study confirmed the importance of raw milk as a possible source of Arcobacter spp. for humans.
\end{abstract}

\section{Introduction}

The Arcobacter genus has been linked to animal and human illness. The species Arcobacter butzleri, Arcobacter cryaerophilus and Arcobacter skirrowii have been associated with several cases of gastrointestinal disease in humans. Arcobacter spp. have been isolated from faeces of dairy animals and found to contaminate different foods of animal origin including milk. In fact, ingestion of Arcobacter-contaminated water and food is considered as the most probable route of transmission. In industrialized countries, the most important source of human Arcobacter infection is the consumption of raw or undercooked food (Giacometti et al., 2014, 2015a, 2015b; Van Driessche and Houf, 2008). Because of the lack of standardized method for the isolation of Arcobacter spp., the true prevalence of this potential pathogen in food is little known and probably underestimated (Collado and Figueras, 2011; Hsu and Lee, 2015; Ramees et al., 2017).

Currently, numerous genotyping methods are used for epidemiological molecular studies. Multi-Locus Sequence Typing (MLST) is a widely used technique for the genetic characterization of Arcobacter spp.; this methodology thanks to the availability of online database of all Arcobacter spp. isolated in the world, allows to compare strains resulting from different studies and various geographical areas (Alonso et al., 2014; De Cesare et al., 2015; Merga et al., 2013; Rasmussen et al., 2013). Few studies have investigated the presence of Arcobacter spp. in bovine milk and the prevalence ranged from 1 to $57 \%$ (Ertas et al., 2010; Milesi, 2010; Pianta and Passos, 2007; Revez et al., 2013; Scullion et al., 2006; Serraino et al., 2013a; Shah et al., 2012; Yesilmen et al., 2014). The aim of the present report was to establish the prevalence and characteristics of Arcobacter spp. in bovine bulk tank milk produced in Apulia Region (Italy).

\section{Materials and Methods}

Bovine bulk tank milk samples were collected from 396 dairy farms of Apulia Region. One milk sample per farm was aseptically collected and immediately transported under refrigeration to the laboratory where it was stored at $-80^{\circ} \mathrm{C}$ before testing. Samples were thawed at room temperature and then $10 \mathrm{~mL}$ of milk were homogenized with $90 \mathrm{~mL}$ of Arcobacter Enrichment Broth (Oxoid, Milan, Italy) plus Cefoperazone, Amphotericin B, Teicoplanin (CAT) Selective Supplement (Oxoid) and incubated at $30^{\circ} \mathrm{C}$ for $48 \mathrm{~h}$ in microaerophilic conditions. From each broth, DNA was extracted and subjected to an Arcobacter genus - specific Real Time PCR as described elsewhere (Gonzales et al., 2013). Ten mL of Real Time PCR Arcobacter spp. positive broths were filtered using $0.45 \mu \mathrm{m}$ pore size
Correspondence: Marta Caruso, Istituto Zooprofilattico Sperimentale della Puglia e della Basilicata, Via Manfredonia 20, 71121 Foggia, Italy.

Tel.: +39.0835.386244 - Fax: +39.0835.389559.

E-mail: marta.caruso@izspb.it

Key words: Arcobacter, Milk, Bovine.

Acknowledgements: Study funded by the Italian Ministry of Health. RC IZSPB 006/2014.

Contributions: the authors contributed equally.

Conflict of interest: the authors declare no potential conflict of interests.

Funding: none.

Received for publication: 10 July 2018 .

Revision received: 16 October 2018.

Accepted for publication: 17 October 2018.

This work is licensed under a Creative Commons Attribution-NonCommercial 4.0 International License (CC BY-NC 4.0).

(C) Copyright M. Caruso et al., 2018

Licensee PAGEPress, Italy

Italian Journal of Food Safety 2018; 7:7685

doi:10.4081/ijfs.2018.7685

syringe filters (Sartorius Stedim Biotech $\mathrm{GmbH}$, Germany) and $0.2 \mathrm{~mL}$ of each filtered broth were spread in parallel onto Columbia Blood, Modified Charcoal Cefoperazone Deoxycholate (MCCD) and Karmali Agar plates (Oxoid). Plates were incubated at $30^{\circ} \mathrm{C}$ in microaerophilic conditions and checked daily for 3-4 days. Presumptive identification tests (Gram staining, catalase and oxidase tests) were performed on suspected colonies among those grown on Columbia Blood, MCCD and Karmali Agar plates. Colonies of Gramnegative spiral bacteria, oxidase and catalase positive were considered presumptive Arcobacter spp. and subjected to species identification using an atp $A$ amplification and sequencing method (Miller et al., 2014). Colonies, all identified as $A$. butzleri, were characterized by Multi-Locus Sequence Typing (MLST) performed on seven housekeeping loci ( $\operatorname{ssp} A, \operatorname{atp} A, \operatorname{gln} A$, glt $A$, glyA,pgm and $t k t$ ) according to the protocol published by Miller et al. (Miller et al., 2009). The different sequences were assigned as alleles and the alleles at the seven loci provided an allelic profile or ST. Allele numbers and STs were assigned using the specific MLST scheme (http://pubmlst.org/arcobacter/) (Miller et al., 2009). 


\section{Results}

The Arcobacter genus - specific PCR revealed the presence of Arcobacter spp. in 64 (16\%) of the 396 milk samples analysed. Arcobacter spp. were isolated from 20 (31\%) of the 64 PCR positive samples. Presumptive identification tests performed on suspected colonies always identified Gram-negative spiral bacteria, oxidase and catalase positive. Species identification of Arcobacter spp. presumptive colonies identified $A$. butzleri in all the 20 positive samples. Prevalence of Arcobacter spp. in bovine milk samples was $5 \%$ (20/396). All the 20 A. butzleri isolates were successfully typed by MLST and a large number of alleles and Sequence Types (STs) were recognized (Table 1).

Specifically, 81 alleles were identified across all the seven loci and 15 (19\%) were previously unreported (Table 1). A total of 16 STs were identified among the $20 \mathrm{~A}$. butzleri isolates analysed; overall $14(87 \%)$ of the 16 STs identified were previously unreported and resulted from new alleles' sequences. Moreover, 13 (81\%) of the 16 STs identified were represented by a single isolate and only ST66, ST420 and ST633 by more than one. The most common sequence type was ST66, identified in $3(15 \%)$ of the isolates, followed by ST633 and ST420, both shared by $2(10 \%)$ isolates.

\section{Discussion}

The aim of the present study was to assess the prevalence and characteristics of Arcobacter spp. in bovine bulk tank milk produced in Apulia Region. Prevalence of Arcobacter spp. in bovine milk samples was $5 \%(20 / 396)$. Studies on the presence of Arcobacter spp. in bovine milk have been performed in different countries and different results have been reported (Ertas et al., 2010; Milesi, 2010; Pianta and Passos, 2007; Revez et al., 2013; Scullion et al., 2006; Serraino et al., 2013a; Shah et al., 2012; Yesilmen et al., 2014). Surveys on bulk tank milk reported prevalence rates of $5.8 \%, 15 \%$ and $46 \%$ in Malaysia, Finland and Northern Ireland respectively (Revez et al., 2013; Scullion et al., 2006; Shah et al., 2012). In Italy a prevalence rate of $26 \%$ was found in bulk tank milk produced in Lombardia Region and a study performed on in-line milk filters of dairy farms authorized to produce and sell raw milk for direct human consumption revealed Arcobacter spp. in $57 \%$ of analysed samples (Milesi, 2010; Serraino et al., 2013a). Although the different prevalence rates reported in literature could be due to different sampling methods and to the absence of a standardized protocol of analysis, the detection of very different values in studies performed on the same sample type and using similar protocols could be related to numerous factors such as hygiene of farms, source of water, feeding, climate etc. (Collado and Figueras, 2011; Hsu and Lee, 2015).

A. butzleri was the only isolated species in the bulk tank milk samples analysed, in agreement with the results of previous studies that reported $A$. butzleri as the most commonly recovered species both in milk and milk products, and in dairy plants (Giacometti et al., 2013; Serraino et al., 2013a). The more frequent isolation of $A$. butzleri has been attributed to its ability to grow in several substrates and in different environmental conditions and to survive to sanitizing procedures (Giacometti et al., 2014; Rassmussen et al., 2013).

MLST analysis confirmed a considerable amount of genetic diversity between the $A$. butzleri isolates (Alonso et al., 2014; De Cesare et al., 2015; Merga et al., 2011, 2013; Miller et al., 2009; PérezCataluña et al., 2017; Rasmussen et al., 2013). In fact, 13 (81\%) of the 16 STs identified were represented by a single isolate and only ST66, ST420 and ST633 by more than one. Moreover, $15(19 \%)$ of the 81 alleles and $14(87 \%)$ of the 16 STs identified were previously unreported. A high degree of heterogeneity was demonstrated also by other authors using

Table 1. Origin and MLST typing data of A. butzleri strains isolated.

\begin{tabular}{|c|c|c|c|c|c|c|c|c|c|}
\hline Strain & Source & aspA & atpA & $g \ln A$ & gltA & glyA & pgm & tkt & ST \\
\hline 1 & FARM 27 & 15 & 10 & 1 & 17 & 19 & 2 & 13 & 66 \\
\hline 2 & FARM 34 & 15 & 10 & 1 & 17 & 19 & 2 & 13 & 66 \\
\hline 3 & FARM 38 & 6 & 23 & 1 & 11 & 494 & 58 & 199 & $627^{*}$ \\
\hline 4 & FARM 63 & 48 & 25 & 41 & 19 & 487 & 101 & $272^{*}$ & $633^{*}$ \\
\hline 5 & FARM 64 & 15 & 10 & 1 & 17 & 186 & 102 & 13 & $628 *$ \\
\hline 6 & FARM 70 & 77 & $209^{*}$ & 1 & 17 & $637^{*}$ & $339 *$ & 199 & $634^{*}$ \\
\hline 7 & FARM 155 & 5 & 5 & 9 & 15 & 120 & 7 & 6 & $629 *$ \\
\hline 8 & FARM 166 & 20 & 39 & 34 & 19 & 104 & $340^{*}$ & 51 & $635^{*}$ \\
\hline 9 & FARM 167 & 23 & 17 & 17 & 19 & 461 & 11 & 65 & $630^{*}$ \\
\hline 10 & FARM 184 & 209 & 15 & $186^{*}$ & 48 & $638^{*}$ & 74 & 86 & $646^{*}$ \\
\hline 11 & FARM 227 & 5 & 5 & 5 & 15 & 66 & 11 & 10 & 420 \\
\hline 12 & FARM 241 & $309^{*}$ & $210^{*}$ & 4 & 146 & 467 & 58 & 14 & $636^{*}$ \\
\hline 13 & FARM 242 & 20 & 20 & 11 & 19 & $639 *$ & 255 & 11 & $647^{*}$ \\
\hline 14 & FARM 244 & 13 & 12 & 1 & $208^{*}$ & $640^{*}$ & 290 & 165 & $648^{*}$ \\
\hline 15 & FARM 261 & $310^{*}$ & 133 & 11 & 19 & 19 & 123 & $271^{*}$ & $637^{*}$ \\
\hline 16 & FARM 271 & 20 & 12 & 11 & 19 & 458 & 11 & 10 & $631^{*}$ \\
\hline 17 & FARM 274 & 15 & 10 & 1 & 17 & 19 & 2 & 13 & 66 \\
\hline 18 & FARM 312 & 5 & 5 & 5 & 15 & 66 & 11 & 10 & 420 \\
\hline 19 & FARM 344 & 48 & 25 & 41 & 19 & 487 & 101 & $272^{*}$ & $633^{*}$ \\
\hline 20 & FARM 351 & 17 & 15 & 15 & 12 & 66 & 102 & 17 & $632 *$ \\
\hline
\end{tabular}

*Represent novel alleles or sequence types. 
different genotyping methods such as PFGE (Pulsed-field gel electrophoresis), MLVA (Multiple Locus Variable-number Tandem repeat Analysis), AFLP (Amplified fragment length polymorphism), RAPD (Random Amplification of Polymorphic DNA), and ERIC-PCR (Enterobacterial Repetitive Intergenic Consensus) (De Cesare et al., 2015; Douidah et al., 2014; Ramees et al., 2017). As more than 600 profiles are currently available in the PubMLST database, the high number of STs identified clearly shows the high genetic diversity of A. butzleri, as previously described (Alonso et al., 2014; De Cesare et al., 2015; Miller et al., 2009). The high variability of $A$. butzleri is believed to be due to genetic recombination (Alonso et al., 2014). Furthermore, the high level of heterogeneity among the $A$. butzleri isolates obtained by MLST confirms the high discriminatory power of this method and its suitability for epidemiological investigations.

Although the prevalence of Arcobacter spp. in bovine bulk tank milk from Apulia Region was low, the results of the present study demonstrate that raw milk could be a vehicle of this important zoonotic pathogen. Consumption of milk after heat treatment and the use of pasteurized milk in cheese production are believed to be effective in preventing potential public health risks. About $75 \%$ of raw milk produced in Apulia Region is delivered to artisanal cheese factories that transform milk in traditional dairy products, most of which, such as fresh stretched cheeses (mozzarella, burrata, scamorza etc.) are made from unpasteurized milk (Sottili, personal communication). Considering that it has been demonstrated that Arcobacter spp. is able to survive under the temperature conditions used for the production of many traditional dairy products such as mozzarella cheese (Serraino et al., 2013b), the possible public health implications due to the consumption of raw milk cheese must be seriously evaluated. Besides the risk associated to the consumption of contaminated raw milk and raw milk cheese, it must be also considered that these products could represent a potential source for the secondary contamination of ready to eat foods that are not usually cooked before consumption.

\section{Conclusions}

Although the prevalence of Arcobacter spp. in bovine bulk tank milk from Apulia Region was low, the results of the present study demonstrate that raw milk could be a potential source of this emerging zoonotic pathogen. Considering that most of milk produced in the investigated area is transformed in raw milk cheese, the results of the present study highlight the need for additional data in order to better assess the human health risk arising from the use of unpasteurized milk for the production of fresh and semi-hard cheese.

\section{References}

Alonso R, Girbau C, MartinezMalaxetxebarria I, Fernandez-Astorga A, 2014. Multilocus sequence typing reveals genetic diversity of foodborne Arcobacter butzleri isolates in the North of Spain. Int J Food Microbiol 191:1258.

Collado L, Figueras MJ, 2011. Taxonomy, epidemiology, and clinical relevance of the genus Arcobacter. Clin Microbiol Rev 24:174-92.

De Cesare A, Parisi A, Giacometti F, Serraino A, Piva S, Caruso M, De Santis EP, Manfreda G, 2015. Multilocus sequence typing of Arcobacter butzleri isolates collected from dairy plants and their products, and comparison with their PFGE types. J Appl Microbiol 120:165-74.

Douidah L, De Zutter L, Bare J, Houf, K, 2014. Towards a typing strategy for Arcobacter species isolated from humans and animals and assessment of the in vitro genomic stability. Foodborne Pathog Dis 11:1-9.

Ertas N, Dogruer Y, Gonulalan Z, Guner A, Ulger I, 2010. Prevalence of arcobacter species in drinking water, spring water, and raw milk as determined by multiplex PCR. J Food Prot 73:2099-102.

Giacometti F, Lucchi A, Manfreda G, Florio D, Zanoni RG, Serraino A, 2013. Occurrence and genetic diversity of Arcobacter butzleri in an artisanal dairy plant in Italy. Appl Environ Microbiol 79:6665-9.

Giacometti F, Serraino A, Pasquali F, De Cesare A, Bonerba E, Rosmini R, 2014. Behavior of Arcobacter butzleri and Arcobacter cryaerophilus in ultrahightemperature, pasteurized, and raw cow's milk under different temperature conditions. Foodborne Pathog Dis 11:15-20.

Giacometti F, Lucchi A, Di Francesco A, Delogu M, Grilli E, Guarniero I, Stancampiano L, Manfreda G, Merialdi G, Serraino A, 2015a. Arcobacter butzleri, Arcobacter cryaerophilus, and Arcobacter skirrowii Circulation in a Dairy Farm and Sources of Milk Contamination. Appl Environ Microbiol 81:5055-63.
Giacometti F, Losio MN, Daminelli P, Cosciani-Cunico E, Dalzini E, Serraino A, 2015b. Short communication: Arcobacter butzleri and Arcobacter cryaerophilus survival and growth in artisanal and industrial ricotta cheese. J Dairy Sci 98:6776-81.

Gonzales I, Femandez-Tomè S, Garcia T, Martin R, 2013. Genus-specific PCR assay for screening Arcobacter spp. in chicken meat. J Sci Food Agric 94:121824.

Hsu DT, Lee J, 2015. Global distribution and prevalence of Arcobacter in food and water. Zoonoses Public Health 62:57989.

Merga JY, Leatherbarrow AJ, Winstanley C, Bennett M, Hart CA, Miller WG, Williams NJ, 2011. Comparison of Arcobacter isolation methods, and diversity of Arcobacter spp. in Cheshire, United Kingdom. Appl Environ Microbiol 77:1646-50.

Merga JY, Williams NJ, Miller WG, Leatherbarrow AJ, Bennett M, Hall N, Ashelford KE, Winstanley C, 2013. Exploring the diversity of Arcobacter butzleri from cattle in the UK using MLST and whole genome sequencing. PLoS One 8:e55240.

Milesi S, 2010. Emerging pathogen Arcobacter spp. in food of animal origin. $\mathrm{PhD}$ thesis. Univeristy of Milan, Italy.

Miller WG, Wesley IV, On SI, Houf K, Mègraud F, Wang G, Yee E, Srijan A, Mason CJ, 2009. First multi-locus sequence typing scheme for Arcobacter spp. BMC Microbiol 9:196.

Miller WG, Yee E, Jolley KA, Chapman MH, 2014. Use of an improved atpA amplification and sequencing method to identify members of the Campylobacteraceae and Helicobacteraceae. Lett Appl Microbiol 58:582-90.

Pérez-Cataluña A, Tapiol J, Benavent C, Sarvisé C, Gómez F, Martínez B, TerronPuig M, Recio G, Vilanova A, Pujol I, Ballester F, Rezusta A, Figueras MJ, 2017. Antimicrobial susceptibility, virulence potential and sequence types associated with Arcobacter strains recovered from human faeces. J Med Microbiol 66:1736-43.

Pianta C, Passos DT, 2007. Isolation of Arcobacter spp. from the milk dairy cows in Brazil. Cienc Rural Santa Maria Hepp d 37:171-4.

Ramees TP, Dhama K, Karthik K, Rathore RS, Kumar A, Saminathan M, Tiwari R, Malik YS, Singh RK, 2017. Arcobacter: an emerging food-borne zoonotic pathogen, its public health concerns and advances in diagnosis and control-a comprehensive review. Vet Q 37:136-61. 
Rasmussen LH, Kjeldgaard J, Christensen JP, Ingmer H, 2013. Multilocus sequence typing and biocide tolerance of Arcobacter butzleri from Danish broiler carcasses. BMC Res Notes 6:322.

Revez J, Huuskonen M, Ruusunen M, Lindström M, Hänninen ML, 2013. Arcobacter species and their pulsed-field gel electrophoresis genotypes in Finnish raw milk during summer 2013. J Food Prot 76:1630-2.

Scullion R, Harrington CS, Madden RH, 2006. Prevalence of Arcobacter spp. in raw milk and retail raw meats in Northern Ireland. J Food Prot 69:1986-90.

Serraino A, Florio D, Giacometti F, Piva S,
Mion D, Zanoni RG, 2013a. Presence of Campylobacter and Arcobacter species in in-line milk filters of farms authorized to produce and sell raw milk and of a water buffalo dairy farm in Italy. J Dairy Sci 96:2801-7.

Serraino A, Giacometti F, Daminelli P, Losio MN, Finazzi G, Marchetti G, Zambrini AV, Rosmini R 2013b. Survival of Arcobacter butzleri during production and storage of artisan water buffalo mozzarella cheese. Foodborne Pathog Dis 10:820-4.

Shah AH, Saleha AA, Murugaiyah M, Zunita Z, Memon AA, 2012. Prevalence and distribution of Arcobacter spp. in raw milk and retail raw beef. J Food Prot 75:1474-8.

Sottili R, 2018, personnel communication on "Destination of bovine bulk tank milk in Apulia Region", e-mail, October $10^{\text {th }}$, 2018.

Yesilmen S, Vural A, Erkan ME, Yildirim IH, 2014. Prevalence and antimicrobial susceptibility of Arcobacter species in cow milk, water buffalo milk and fresh village cheese. Int J Food Microbiol 188:11-4.

Van Driessche E and Houf K, 2008. Survival capacity in water of Arcobacter species under different temperature conditions. J Appl Microbiol 105:443-51. 